\title{
Wild gut microbiota protects from disease
}

\author{
Cell Research (2018) 28:135-136. doi:10.1038/cr.2017.150; published online 1 December 2017
}

\section{Wild mice are genetically similar to laboratory mice, but have a distinct gut microbiota that protects from disease.}

There is no doubt that the trillions of bacteria that inhabit the mammalian gut play crucial roles in health and disease. It seems that for every disease or condition, gut bacteria influence the outcome. Therefore, many scientists are dedicated to finding the good bacteria that make our bodies healthier. We control as many environmental factors as we can to try to determine how one bacteria influences a certain condition. However, in doing so, we eliminate the natural variation that occurs outside of the laboratory and potentially overlook important factors that influence host-microbe interactions. In a recent paper published in Cell, Rosshart et al. [1] investigate the genetic and microbial differences between laboratory mice and wild mice and demonstrate that these differences have a profound effect on disease.

What did you do today before you sat down to read this article? Maybe you took the subway into work and touched the rail that thousands of other people touched before you. Maybe your toddler sneezed in your face as you dropped him off at daycare. Or did you run out of eggs this morning and settled for a piece of toast instead? When's the last time you changed your sheets? Not only do diet, exercise, sleep, and hygiene affect our well-being, they also influence our gut microbiota.

Now let's compare our morning to that of laboratory mice. After a night of eating the same food they eat every day, mice start to curl up in their clean beds (changed every two weeks). At 7 am the lights go on, at $7 \mathrm{pm}$ the lights go off. The temperature (though a little colder than ideal) is constant all year long. The mice are routinely tested to make sure they are clear of known pathogens. They are never overcrowded and are rarely housed alone. This controlled environment insures that the mice are well cared for and minimizes environmental factors influencing experimental outcomes. Mice are healthy in these conditions, but what about their bacteria? Are their gut microbes similar to mice in the real world? Does laboratory microbiota translate to human microbiomes?

To answer these questions, Rosshart et al. [1] investigated the genetic composition of wild mice and the makeup of their gut microbiota. They trapped more than 800 wild mice in different barns throughout Maryland and the District of Columbia. Genetic analysis revealed that Maryland mice were actually most similar to laboratory strains of mice compared to wild mice from around the world. The makeup of their microbiota, however, was significantly different. Laboratory mouse microbiota is composed mainly of Bacteroidetes and Firmicutes. The dominant phyla in wild mouse microbiota, on the other hand, were found to be Proteobacteria and Bacteroidetes. Furthermore, wild mouse microbiota contained more species than lab mouse microbiota. While surprising, the current knowledge about gut microbiota does not allow us to predict the effects of the dramatic difference between wild and lab microbiota from this level of taxonomic information.

At the phylum level, laboratory mice and humans share a similar microbiota dominated by Bacteroidetes and Fir- micutes [2]. However, at the species and strain levels, there is very little naturally occurring overlap between laboratory mice and human microbiota [3]. Therefore, to study the effects of human gut bacteria in health and disease, germ-free or antibiotic-treated mice are colonized with human bacteria. Many of these studies have revealed that both Firmicutes and Bacteroidetes can modulate the immune system to promote or protect from disease. For example, both the Bacteriodetes, Bacteroides fragilis, and a mix of Clostridia (Firmicutes) can induce regulatory $\mathrm{T}$ cells, indicating cross-phyla redundancy in the microbiota $[4,5]$. On the other hand, a recent study showed that different strains of the same species can exert different or even opposing effects [6]. For example, $B$. fragilis strain 9343 can protect against colitis [4], whereas B. fragilis strain 865443-2-2 exacerbates colitis in mice [7].

To determine the functional differences between wild and lab microbiota, Rosshart et al. colonize germ-free mice with either laboratory mouse microbiota (referred to as LabR mice) or wild mouse microbiota (WildR mice). When challenged with Influenza A Virus (IAV), only $17 \%$ of LabR mice survived, yet $92 \%$ of WildR mice survived, suggesting that a wild microbiota protects from IAV. Further analysis of these mice showed that WildR mice had lower viral titers in their lungs and a lower pro-inflammatory cytokine response compared to LabR mice. Similarly, in a colon cancer model, WildR mice had fewer, smaller tumors, less inflammation, and fewer metastases than LabR mice. Furthermore, the authors sequenced wild mouse microbiota and 
found that many wild mice were colonized with known pathogens, but had no disease histology, suggesting that wild mouse microbiota promotes tolerance to certain pathogens. As summarized in Figure 1, this study shows that the WildR mouse microbiota enhances fitness and that environmental factors in the real world shape a healthier gut microbiota than what exists in the laboratory. Because the WildR microbiota contains not only a broader diversity of microbes, but also a naturally selected diversity, the authors suggest that using

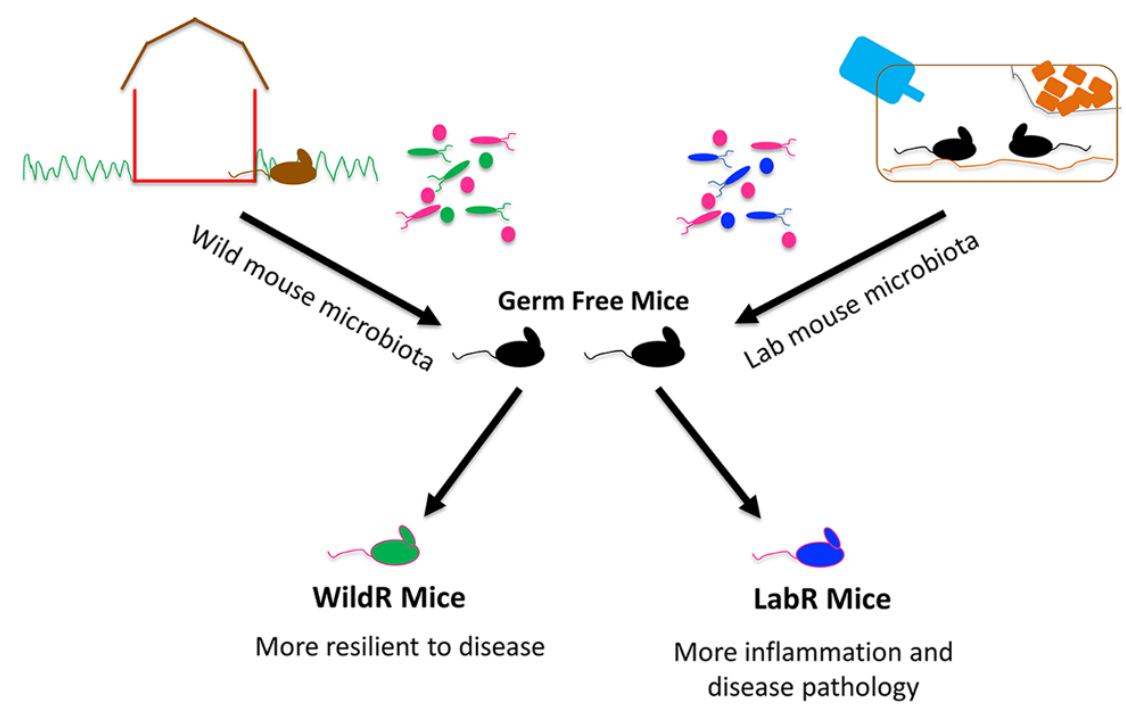

Figure 1 Wild mouse microbiota is dramatically different from laboratory mouse microbiota in both phylogeny and function. Pregnant germ-free mice were colonized with either laboratory mouse microbiota or wild mouse microbiota and their offspring were challenged with Influenza A Virus (IAV) or were subjected to colon cancer induction. Mice with wild mouse microbiota (WildR) were protected from IAV-induced mortality and had reduced tumor burden compared to mice with laboratory mouse microbiota (LabR).
WildR mice for disease models might better represent disease pathology and responses. Importantly, this paper highlights a dramatic functional difference between wild and laboratory microbiota and forces us to deeply consider the best models for studying gut microbe-host interactions.

Francesca S Gazzaniga ${ }^{1}$, Dennis L Kasper ${ }^{1}$

${ }^{1}$ Department of Microbiology and Immunobiology, Harvard Medical School, Boston, MA 02115, USA

Correspondence: Dennis L Kasper

E-mail: dennis_kasper@hms.harvard.edu

\section{References}

1 Rosshart SP, Vassallo BG, Angeletti D, et al. Cell 2017; 171:1015-1028.

2 Eckburg PB, Bik EM, Bernstein CN, et al. Science 2005; 308:1635-1638.

3 Chung H, Pamp SJ, Hill JA, et al. Cell 2012; 149:1578-1593.

4 Mazmanian SK, Round JL, Kasper DL. Nature 2008; 453:620-625.

5 Atarashi K, Tanoue T, Oshima K, et al. $\mathrm{Na}$ ture 2013; 500:232-236.

6 Geva-Zatorsky N, Sefik E, Kua L, et al. Cell 2017; 168:928-943.

7 Rabizadeh S, Rhee KJ, Wu S, et al. Inflamm Bowel Dis 2007; 13:1475-1483. 